\title{
Supplier's Joint Investments in Cost Reduction and Quality Improvement in a Decentralized Supply Chain
}

\author{
Hengyun Zhang ${ }^{1,2}$ and Dingjun Hong ${ }^{1}$ \\ ${ }^{1}$ School of Management and Economics, University of Electronic Science and Technology of China, Chengdu, Sichuan 611731, China \\ ${ }^{2}$ Department of Information Engineering, Sichuan Staff University of Science and Technology, Chengdu, Sichuan 610101, China \\ Correspondence should be addressed to Hengyun Zhang; zhyuestc@163.com
}

Received 27 April 2017; Revised 24 July 2017; Accepted 25 July 2017; Published 22 August 2017

Academic Editor: Vladimir Turetsky

Copyright (c) 2017 Hengyun Zhang and Dingjun Hong. This is an open access article distributed under the Creative Commons Attribution License, which permits unrestricted use, distribution, and reproduction in any medium, provided the original work is properly cited.

\begin{abstract}
We consider a decentralized supply chain with a downstream manufacturer and an upstream supplier. The upstream supplier sells a product to the manufacturer, who faces a quality and price sensitive demand. The supplier has a chance to invest in both cost reduction and quality improvement of its product. We derive the optimal investment and pricing decisions for the supply chain members. We do so in both the centralized and the decentralized supply chains. We show that the optimal investment and pricing decisions in the decentralized supply chain may deviate from that in the centralized supply chain. We develop a mechanism to coordinate the decentralized supply chain. The developed mechanism contains four policies: wholesale price, sharing of revenue, sharing of cost reduction investment cost, and sharing of quality improvement investment cost. We also show that the developed coordination mechanism can lead to Pareto improvement.
\end{abstract}

\section{Introduction}

Low production cost and high product quality are key components in achieving competitive advantage. In reality, many firms have made investments to reduce their production costs and improve their product quality, to create competitive advantages in the increasingly volatile marketplace. For example, in automotive industry, it is quite common that component suppliers engage not only in cost reduction activity and but also in quality improvement activity [1]. In computer industry, many computer manufacturers make investments in both cost reduction and quality improvement [2]. Moreover, empirical studies show that IT investment enables a firm to achieve both cost reduction and quality improvement [3].

There is extensive research on cost reduction investment and quality improvement investment in supply chains. The existing research supposes that a supply chain player makes investment in either quality improvement or cost reduction. For example, $\mathrm{Xu}$ [4] considers a supply chain where the upstream firm makes quality improvement investment.
Xie et al. [5] analyze quality improvement investment of a risk-averse upstream firm. Chao et al. [6] explore quality improvement investments of both the upstream and the downstream firms. Seifbarghy et al. [7] investigate the upstream firm's quality improvement investment in a supply chain where customer demands are divided into two categories. Chen et al. [8] consider quality improvement investment in a dual-channel supply chain where the product can be delivered through both the direct and the retail channels. $\mathrm{Yu}$ and $\mathrm{Ma}$ [9] and Li and Chen [10] study quality improvement investments in a supply chain with multiple upstream firms and one downstream firm. Chen et al. [11] study quality improvement investments in a supply chain with one upstream firm and multiple downstream firms. Zhu et al. [12] and Ma et al. [13, 14] coordinate a supply chain, where the upstream firm conducts quality improvement investment. Yang and Xiao [15] develop a coordination mechanism for a supply chain where consumers are loss-averse in service quality.

There is also some research on cost reduction investment in supply chains. For example, in a supply chain with one 
upstream firm and one downstream firm, Usta et al. [16] consider the downstream firm's cost reduction investment. Ge et al. [17] consider cost reduction investments of both the upstream and the downstream firms. In a supply chain with one upstream firm and multiple downstream firms, Banerjee and Lin [18] investigate the upstream firm's cost reduction investment. Banerjee and Lin [19] investigate cost reduction investments of downstream firms. In a supply chain consisting of multiple upstream firms and one downstream firm, Bernstein and Kök [20] and Bernstein et al. [21] study cost reduction investments of upstream firms. Fu et al. [22] investigate the problem of the downstream firm's cost reduction investment in upstream firms. Additionally, $\mathrm{Mu}$ and Cui [23] study the cost reduction problem in a supply chain by using the experimental approach and put forward theoretical model trust and incentive as the two kinds of interaction of the important mechanism in supply chain cost management. For more detailed discussions of cost reduction management in supply chains, see, for example, Ishii [24] and Li et al. [25].

Although the above researches investigate the problem of cost reduction investment and quality improvement investment in supply chains from different perspectives, they assume that a chain member either conducts cost reduction investment or carries out quality improvement investment. No research investigates the problem of joint investments in cost reduction and quality improvement. Motivated by the gap that exists between theory and practice, this paper considers a supply chain where the downstream manufacturer purchases a product from the upstream supplier and then sells it to a quality and price sensitive market. The supplier has a chance to invest not only in cost reduction, but also in quality improvement. We first derive the optimal pricing decisions under any given investment strategy. Then, we characterize the optimal cost reduction and quality improvement investment strategies. We consider both the centralized and the decentralized supply chains. Finally, we develop a mechanism to achieve supply chain coordination.

The main contributions of our paper can be summarized as follows:

(1) We develop a model to study the problem of the supplier's joint investments in cost reduction and quality improvement in a supply chain environment.

(2) We characterize the optimal pricing decisions in both the centralized and the decentralized supply chains, under any given investment strategy.

(3) We derive the optimal investment strategies in both the centralized and the decentralized supply chains and obtain many interesting managerial insights.

(4) We develop a mechanism to coordinate the decentralized supply chain and show that the developed coordination mechanism can result in Pareto improvement.

The rest of this paper is structured as follows. In Section 2, we describe the model. In Section 3, we study investment and pricing decisions in the centralized supply chain. Section 4 analyzes the decisions in decentralized supply chain.
Section 5 addresses the problem of supply chain coordination. Section 6 concludes the paper and outlines future works.

\section{The Model}

Consider a supply chain consisting of an upstream supplier and a downstream manufacturer. The supplier provides a product to the manufacturer, and then the manufacturer sells it to the market. The intrinsic production cost is $c$, and the wholesale price of the supplier's product is $w$.

The supplier has a chance to make investments not only in cost reduction, but also in quality improvement. We denote the reduction level in the supplier's production cost and the improvement level in the product quality, caused by the investments, as $x$ and $y$, respectively, and the costs of cost reduction investment and quality improvement investment as $d(x)$ and $e(y)$, respectively. Similar to d'Aspremont and Jacquemin [26] and Ge (2014), we assume that $d(x)$ is convexly increasing in $x$ and takes the form of

$$
d(x)=I x^{2}
$$

where $I$ in (1) is a parameter related to the marginal cost of cost reduction investment and $I>0$. Moreover, similar to Ghosh and Shah [27] and Ma et al. [13, 14], we assume that $e(y)$ is convexly increasing in $y$ and takes the form of

$$
e(y)=J y^{2}
$$

where $J$ in (2) is a parameter related to the marginal cost of quality improvement investment and $J>0$. We further assume that the supplier's investment activity is constrained by $0 \leq I x^{2}+J y^{2} \leq K$, in the sense that the total amount of resources that can be invested is limited. To ensure that the supplier's production cost is always positive, that is, $c-x>0$, we require $I>K / c^{2}$.

Customer demand $D(p, y)$ faced by the manufacturer is quality and price sensitive. Without loss of generality, we assume that the customer demand function is given by

$$
D(p, y)=a-b p+\beta y,
$$

where $a$ in (3) is the potential intrinsic customer demand, $p$ corresponds to the retail price of the product, $y$ corresponds to the quality level of the product, $b$ represents the demand sensitivity to the retail price, and $\beta$ represents the demand sensitivity to the product quality (see, e.g., $[5,28]$ ).

The decisions to be made by the supplier and the manufacturer fall into three stages:

(i) In the first stage, the supplier chooses its investment strategy $(x, y)$; that is, it determines the values for $x$ and $y$, respectively.

(ii) In the second stage, the supplier chooses the wholesale price $w$ for the product to be charged to the manufacturer.

(iii) In the last stage, the manufacturer determines the retail price $p$ for the product. 
Both the supplier and the manufacturer are risk-neutral, in the sense that they will choose their decisions to maximize their own profits. Based upon the above description, we can obtain the profit functions of the supplier and the manufacturer, respectively, as follows:

$$
\begin{aligned}
\Pi_{s}(w \mid x, y)= & {[w-(c-x)](a-b p+\beta y)-I x^{2} } \\
& -J y^{2}, \\
\Pi_{m}(p \mid x, y)= & (p-w)(a-b p+\beta y) .
\end{aligned}
$$

\section{Centralized Supply Chain}

To provide a benchmark for the decentralized supply chain, we first study the decision problem in the centralized supply chain, where the manufacturer and the supplier make decisions to maximize the entire supply chain's profit, that is, the total profit of the manufacturer and the supplier.

It follows from (4) and (5) that the profit of the entire supply chain can be written as

$$
\begin{aligned}
\Pi_{c}(p \mid x, y)= & \Pi_{s}(w \mid x, y)+\Pi_{m}(p \mid x, y) \\
= & {[p-(c-x)](a-b p+\beta y)-I x^{2} } \\
& -J y^{2} .
\end{aligned}
$$

Note from (6) that the wholesale price $w$ vanishes. This is because $w q$ is the internal transfer payment between the supplier and the manufacturer. Thus, in the centralized supply chain, the decision problem is to choose values for $x, y$, and $p$. We will first consider the decision on the retail price $p$, assuming that investment strategy $(x, y)$ is given, and then investigate the investment strategy.

From (6), we have the following lemma.

Lemma 1. For any given investment strategy $(x, y)$, the optimal retail price $p_{c}^{*}(x, y)$ for the centralized supply chain is

$$
p_{c}^{*}(x, y)=\frac{a+\beta y+b(c-x)}{2 b} .
$$

Proof of Lemma 1. Taking the first and the second derivatives of $\Pi_{c}(p \mid x, y)$ of $(6)$ with respect to $p$, we have

$$
\begin{aligned}
& \frac{d \Pi_{c}(p \mid x, y)}{d p}=a+b(c-x)-2 b p+\beta y, \\
& \frac{d^{2} \Pi_{c}(p \mid x, y)}{d p^{2}}=-2 b .
\end{aligned}
$$

That is, the profit of the entire supply chain is strictly concave in the retail price. Thus, the optimal retail price for the centralized supply chain satisfies the first-order condition; that is, $d \Pi_{c}(p \mid x, y) / d p=0$. Solving the first-order condition for $p$, we get (7). We thus complete the proof of Lemma 1.

Lemma 1 characterizes the optimal retail price, under any given investment strategy. From Lemma 1, we know that the retail price decreases as the cost reduction $x$ increases. That is, a lower retail price will be set for the product with a lower production cost. Moreover, Lemma 1 also states that the retail price increases in the product quality $y$. That is, a higher retail price will be set for the product with a higher product quality. The above insights are rather intuitive.

Next, we consider the optimal investment strategy in the centralized supply chain. Substituting $p=p_{c}^{*}(x, y)$ of (7) into $\Pi_{c}(p \mid x, y)$ of $(6)$, the problem of investment strategy $(x, y)$ can be written as

$$
\begin{aligned}
\max _{x, y} & \Pi_{c}(x, y)=\frac{[a+\beta y-b(c-x)]^{2}}{4 b}-I x^{2}-J y^{2}, \\
\text { s.t. } \quad & x \geq 0, \\
& y \geq 0, \\
& 0 \leq I x^{2}+J y^{2} \leq K,
\end{aligned}
$$

which is a two-dimensional constrained optimization problem. The following theorem provides a necessary condition for the optimal investment strategy.

Theorem 2. For any given total amount of investment resources $I x^{2}+J y^{2}$, denoted by $k, 0 \leq k \leq K$, the entire supply chain's profit function $\Pi_{c}(x, y)$ of (9) achieves its maximum at

$$
\begin{aligned}
& x_{c}^{*}(k)=\frac{J^{1 / 2} b}{\left(I^{2} \beta^{2}+I J b^{2}\right)^{1 / 2}} \cdot k^{1 / 2}, \\
& y_{c}^{*}(k)=\frac{I J^{-1 / 2} \beta}{\left(I^{2} \beta^{2}+I J b^{2}\right)^{1 / 2}} \cdot k^{1 / 2} .
\end{aligned}
$$

Proof of Theorem 2. For any given $I x^{2}+J y^{2}=k$, we have $y=\left(k-I x^{2}\right)^{1 / 2} / J^{1 / 2}$, and thus the entire supply chain's profit function $\Pi_{c}(x, y)$ of $(9)$ can be rewritten as

$$
\Pi_{c}(x)=\frac{\left[a+\beta\left(k-I x^{2}\right)^{1 / 2} / J^{1 / 2}-b(c-x)\right]^{2}}{4 b}-k .
$$

Clearly, for any given $I x^{2}+J y^{2}=k$, maximizing $\Pi_{c}(x)$ is equivalent to maximizing

$$
M(x)=a+\frac{\beta\left(k-I x^{2}\right)^{1 / 2}}{J^{1 / 2}}-b(c-x) .
$$

Taking the first derivative of $M(x)$ with respect to $x$, we have

$$
\frac{d M(x)}{d x}=b-\frac{\beta I}{J^{1 / 2}\left(k / x^{2}-I\right)^{1 / 2}},
$$

which is decreasing in $x$ and indicates that $M(x)$ is strictly concave in $x$. With this, in conjunction with the facts that $x \geq 0, I x^{2} \leq k, \lim _{x \rightarrow 0}(d M(x) / d x)>0$, and $\lim _{x \rightarrow\left(k^{1 / 2} / I^{1 / 2}\right)}(d M(x) / d x)<0$, we know that the maximizer of $M(x)$ is uniquely determined by the first-order condition; that is, $d M(x) / d x=0$. Solving the first-order condition for 
$x$, we get $x=x_{c}^{*}(k)$ of (10). Substituting $x=x_{c}^{*}(k)$ of (10) into $I x^{2}+J y^{2}=k$, we have $y=y_{c}^{*}(k)$ of (11). We thus complete the proof of Theorem 2 .

Theorem 2 shows that the optimal investment strategy for the entire supply chain requires that the resources be invested not only in cost reduction, but also in quality improvement. After some algebra, we can show that, for any given total amount of investment resources $I x^{2}+J y^{2}=k$, the cost reduction investment strategy and the quality improvement investment strategy are, respectively, as follows:

$$
\begin{aligned}
& I\left(x_{c}^{*}(k)\right)^{2}=\frac{I J b^{2}}{I^{2} \beta^{2}+I J b^{2}} \cdot k \\
& J\left(y_{c}^{*}(k)\right)^{2}=\frac{I^{2} \beta^{2}}{I^{2} \beta^{2}+I J b^{2}} \cdot k
\end{aligned}
$$

implying that the ratio of resources invested in cost reduction and quality improvement in the centralized supply chain is

$$
\frac{I\left(x_{c}^{*}(k)\right)^{2}}{J\left(y_{c}^{*}(k)\right)^{2}}=\frac{J b^{2}}{I \beta^{2}} .
$$

Equation (17) states that when the parameter $I$ (or the parameter $\beta$ ) is relatively large, more resources should be invested to improve the product quality; when the parameter $J$ (or the parameter $b$ ) is relatively large, more resources should be invested to reduce the production cost. Second, in conjunction with $x_{c}^{*}(k)$ of (10) and $y_{c}^{*}(k)$ of (11), we can reduce the two-dimensional constrained optimization problem of (9) to the following one-dimensional constrained optimization problem:

$$
\begin{array}{ll}
\max _{k} & \Pi_{c}(k)=\frac{\left[a-b c+I J^{-1 / 2} \beta^{2} /\left(I^{2} \beta^{2}+I J b^{2}\right)^{1 / 2} \cdot k^{1 / 2}+J^{1 / 2} b^{2} /\left(I^{2} \beta^{2}+I J b^{2}\right)^{1 / 2} \cdot k^{1 / 2}\right]^{2}}{4 b}-k \\
\text { s.t. } \quad 0 \leq k \leq K .
\end{array}
$$

That is, one can first determine the total amount of investment resources, that is, $k=I x^{2}+J y^{2}$, and then choose $x$ and $y$ according to $x_{c}^{*}(k)$ of $(10)$ and $y_{c}^{*}(k)$ of (11), respectively.

We are now ready to establish the following theorem.

Theorem 3. The entire supply chain's profit function $\Pi_{c}(k)$ of (18) is strictly concave in $k, 0 \leq k \leq K$, and the optimal total amount of investment resources in the centralized supply chain is given by

$$
k_{c}^{*}= \begin{cases}\bar{k}, & \text { if } \bar{k}<K, \\ K, & \text { if } \bar{k} \geq K,\end{cases}
$$

where

$$
\begin{aligned}
& \bar{k} \\
& =\left[\frac{(a-b c)\left(I J^{-1 / 2} \beta^{2}+J^{1 / 2} b^{2}\right)\left(I^{2} \beta^{2}+I J b^{2}\right)^{1 / 2}}{4 b\left(I^{2} \beta^{2}+I J b^{2}\right)-\left(I J^{-1 / 2} \beta^{2}+J^{1 / 2} b^{2}\right)^{2}}\right]^{2} .
\end{aligned}
$$

Consequently, the optimal investment strategy in the centralized supply chain is given by

$$
\begin{aligned}
& x_{c}^{*}=\frac{J^{1 / 2} b}{\left(I^{2} \beta^{2}+I J b^{2}\right)^{1 / 2}} \cdot\left(k_{c}^{*}\right)^{1 / 2}, \\
& y_{c}^{*}=\frac{I J^{-1 / 2} \beta}{\left(I^{2} \beta^{2}+I J b^{2}\right)^{1 / 2}} \cdot\left(k_{c}^{*}\right)^{1 / 2} .
\end{aligned}
$$

Proof of Theorem 3. Taking the first derivative of $\Pi_{c}(k)$ of (18) with respect to $k$, we have, after some algebra, that

$$
\begin{aligned}
& \frac{d \Pi_{c}(k)}{d k} \\
& =\frac{I J^{-1 / 2} \beta^{2}+J^{1 / 2} b^{2}}{4 b\left(I^{2} \beta^{2}+I J b^{2}\right)^{1 / 2}}\left[\frac{I J^{-1 / 2} \beta^{2}+J^{1 / 2} b^{2}}{\left(I^{2} \beta^{2}+I J b^{2}\right)^{1 / 2}}+\frac{a-b c}{k^{1 / 2}}\right] \\
& \quad-1 .
\end{aligned}
$$

Clearly, $d \Pi_{c}(k) / d k$ is decreasing in $k$, and thus $\Pi_{c}(k)$ is strictly concave in $k$. Consequently, the maximizer of profit function $\Pi_{c}(k)$ is uniquely determined by the first-order condition; that is, $d \Pi_{c}(k) / d k=0$. Solving the first-order condition for $k$, we can get $\bar{k}$ of (20). This, in conjunction with $0 \leq k \leq K$, indicates $k_{c}^{*}$ of (19). Substituting $k=k_{c}^{*}$ of (19) into $x=x_{c}^{*}(k)$ of (10) and $y=y_{c}^{*}(k)$ of (11), respectively, we can obtain $x_{c}^{*}$ of (21) and $y_{c}^{*}$ of (22), respectively. We thus complete the proof of Theorem 3.

Theorem 3 characterizes the optimal investment strategy $\left(x_{c}^{*}, y_{c}^{*}\right)$ for the centralized supply chain, which depends on supply chain parameters, such as $a, b, \beta, I, J$, and $K$.

\section{Decentralized Supply Chain}

In this section, we explore the decentralized supply chain, where the supplier and the manufacturer are independent decision-makers, and choose their optimal decisions by engaging in a three-stage game. We analyze the decision problem, using a three-stage approach. 
4.1. The Third Stage. Assuming that the supplier's investment and wholesale price decisions are given, the manufacturer determines a retail price $p$ for the product to maximize its profit.

From (5), we have the following lemma.

Lemma 4. For any investment strategy $(x, y)$ and wholesale price $w$ chosen by the supplier, the manufacturer's profit function $\Pi_{m}(p \mid x, y)$ of (5) is strictly concave in $p$, and the optimal retail price is given by

$$
p^{*}(w, x, y)=\frac{a+b w+\beta y}{2 b}
$$

Proof of Lemma 4. Taking the first and the second derivatives of $\Pi_{m}(p \mid x, y)$ of (5) with respect to $p$, we have

$$
\begin{aligned}
& \frac{d \Pi_{m}(p \mid x, y)}{d p}=a+b w-2 b p+\beta y, \\
& \frac{d^{2} \Pi_{m}(p \mid x, y)}{d p^{2}}=-2 b .
\end{aligned}
$$

That is, the profit of the manufacturer is strictly concave in the retail price. Thus, the optimal retail price for the manufacturer satisfies the first-order condition; that is, $d \Pi_{m}(p$ । $x, y) / d p=0$. Solving the first-order condition for $p$, we get (24). We thus complete the proof of Lemma 4.

Lemma 4 gives the manufacturer's best response retail price $p^{*}(w, x, y)$. From Lemma 4 , we can see that the response retail price increases not only in wholesale price $w$, but also in product quality $y$. That is, the downstream manufacturer with a higher procurement cost (i.e., wholesale price) will set a higher retail price for the product, and the downstream manufacturer will also set a higher retail price for the product with a higher quality.

4.2. The Second Stage. Knowing that the downstream manufacturer sets the retail price $p^{*}(w, x, y)$ according to (24), the upstream supplier chooses a wholesale price $w$ to maximize its profit, in the second stage. By substituting $p^{*}(w, x, y)$ of (24) into (4), we can rewrite the profit function of the supplier as follows:

$$
\begin{aligned}
\Pi_{s}(w \mid x, y)= & \frac{[w-(c-x)](a+\beta y-b w)}{2}-I x^{2} \\
& -J y^{2} .
\end{aligned}
$$

This allows us to get the following lemma.

Lemma 5. For any given investment strategy $(x, y)$, the supplier's profit function $\Pi_{s}(w \mid x, y)$ of (26) is strictly concave in $w$, and the optimal wholesale price is given by

$$
w^{*}(x, y)=\frac{a+\beta y+b(c-x)}{2 b} .
$$

Proof of Lemma 5. Taking the first and the second derivatives of $\Pi_{s}(w \mid x, y)$ of (26) with respect to $w$, we have

$$
\begin{aligned}
& \frac{d \Pi_{s}(w \mid x, y)}{d w}=\frac{a-2 b w+\beta y+b(c-x)}{2}, \\
& \frac{d^{2} \Pi_{s}(w \mid x, y)}{d w^{2}}=-b
\end{aligned}
$$

That is, the supplier's profit function is strictly concave in the wholesale price. Thus, the wholesale price for the supplier satisfies the first-order condition; that is, $d \Pi_{s}(w \mid x, y) / d w=$ 0 . Solving the first-order condition for $w$, we get (27). We thus complete the proof of Lemma 5.

Lemma 5 gives the supplier's best response wholesale price $w^{*}(x, y)$. From Lemma 5, we can see that the response wholesale price decreases in the production cost $c-x$ (i.e., the response wholesale price $w^{*}(x, y)$ increases in the cost reduction $x$ ) and increases in the product quality $y$. That is, the supplier with a lower production cost will set a lower wholesale price for the product, and the supplier will set a higher wholesale price for the product with a higher quality.

4.3. The First Stage. Anticipating the best response retail price $p^{*}(w, x, y)$ of $(24)$ and the best response wholesale price $w^{*}(x, y)$ of $(27)$, the supplier chooses its investment strategy to maximize its profit in the first stage. Substituting $w^{*}(x, y)$ of (27) into $\Pi_{s}(w \mid x, y)$ of (26), the problem of the supplier's investment strategy $(x, y)$ can be expressed as the following two-dimensional constrained optimization problem:

$$
\begin{aligned}
\max _{x, y} & \Pi_{s}(x, y)=\frac{[a+\beta y-b(c-x)]^{2}}{8 b}-I x^{2}-J y^{2}, \\
\text { s.t. } \quad & x \geq 0 \\
& y \geq 0 \\
& 0 \leq I x^{2}+J y^{2} \leq K .
\end{aligned}
$$

This enables us to derive the following theorem.

Theorem 6. For any given total amount of investment resources $k=I x^{2}+J y^{2}, 0 \leq k \leq K$, the supplier's profit function $\Pi_{s}(x, y)$ of (29) achieves its maximum at

$$
\begin{aligned}
& x^{*}(k)=\frac{J^{1 / 2} b}{\left(I^{2} \beta^{2}+I J b^{2}\right)^{1 / 2}} \cdot k^{1 / 2}, \\
& y^{*}(k)=\frac{I J^{-1 / 2} \beta}{\left(I^{2} \beta^{2}+I J b^{2}\right)^{1 / 2}} \cdot k^{1 / 2} .
\end{aligned}
$$

Proof of Theorem 6. For any given $k=I x^{2}+J y^{2}$, we have $y=\left(k-I x^{2}\right)^{1 / 2} / J^{1 / 2}$, and hence the supplier's profit function $\Pi_{s}(x, y)$ of $(29)$ can be rewritten as

$$
\Pi_{s}(x)=\frac{\left[a+\beta\left(k-I x^{2}\right)^{1 / 2} / J^{1 / 2}-b(c-x)\right]^{2}}{8 b}-k .
$$


Clearly, for any given $k=I x^{2}+J y^{2}$, maximizing $\Pi_{s}(x)$ is equivalent to maximizing

$$
M(x)=a+\frac{\beta\left(k-I x^{2}\right)^{1 / 2}}{J^{1 / 2}}-b(c-x) .
$$

In conjunction with $M(x)$ analyzed in the proof of Theorem 2, we can get $x^{*}(k)$ of $(30)$ and $y^{*}(k)$ of (31). We thus complete the proof of Theorem 6 .

Theorem 6 shows that the optimal investment strategy for the supplier in the decentralized supply chain requires that the resources be invested in both the cost reduction and the quality improvement. Comparing Theorem 6 with Theorem 2, we can conclude that the ratios of resources invested to achieve cost reduction and quality improvement are identical in the centralized and decentralized supply chains; that is, $I\left(x^{*}\right)^{2} / J\left(y^{*}\right)^{2}=I\left(x_{c}^{*}\right)^{2} / J\left(y_{c}^{*}\right)^{2}$. Consequently, the supplier can first decide the total amount of investment resources for production cost reduction and product quality improvement, that is, $k=I x^{2}+J y^{2}$, and then set $x$ and $y$ according to $x^{*}(k)$ of $(30)$ and $y^{*}(k)$ of $(31)$, respectively.

Based upon the above description, we can reduce the problem of the supplier's investment strategy $(x, y)$ to the following one-dimensional constrained optimization problem:

$$
\begin{array}{ll}
\max _{k} & \Pi_{s}(k)=\frac{\left[a-b c+I J^{-1 / 2} \beta^{2} /\left(I^{2} \beta^{2}+I J b^{2}\right)^{1 / 2} \cdot k^{1 / 2}+J^{1 / 2} b^{2} /\left(I^{2} \beta^{2}+I J b^{2}\right)^{1 / 2} \cdot k^{1 / 2}\right]^{2}}{8 b}-k \\
\text { s.t. } & 0 \leq k \leq K .
\end{array}
$$

This leads to the following theorem.

Theorem 7. The supplier's profit function $\Pi_{s}(k)$ of (34) is strictly concave in $k, 0 \leq k \leq K$, and the optimal total amount of investment resources for the supplier in the decentralized supply chain is

$$
k^{*}= \begin{cases}\overline{\bar{k}}, & \text { if } \overline{\bar{k}}<K, \\ K, & \text { if } \overline{\bar{k}} \geq K,\end{cases}
$$

where

$$
\begin{aligned}
& \overline{\bar{k}} \\
& =\left[\frac{(a-b c)\left(I J^{-1 / 2} \beta^{2}+J^{1 / 2} b^{2}\right)\left(I^{2} \beta^{2}+I J b^{2}\right)^{1 / 2}}{8 b\left(I^{2} \beta^{2}+I J b^{2}\right)-\left(I J^{-1 / 2} \beta^{2}+J^{1 / 2} b^{2}\right)^{2}}\right]^{2} .
\end{aligned}
$$

Consequently, the optimal investment strategy for the supplier in the decentralized supply chain is

$$
\begin{aligned}
& x^{*}=\frac{J^{1 / 2} b}{\left(I^{2} \beta^{2}+I J b^{2}\right)^{1 / 2}} \cdot\left(k^{*}\right)^{1 / 2}, \\
& y^{*}=\frac{I J^{-1 / 2} \beta}{\left(I^{2} \beta^{2}+I J b^{2}\right)^{1 / 2}} \cdot\left(k^{*}\right)^{1 / 2} .
\end{aligned}
$$

Proof of Theorem 7. Taking the first derivative of $\Pi_{s}(k)$ of (34) with respect to $k$, we have, after some algebra, that

$$
\begin{aligned}
& \frac{d \Pi_{s}(k)}{d k} \\
& =\frac{I J^{-1 / 2} \beta^{2}+J^{1 / 2} b^{2}}{8 b\left(I^{2} \beta^{2}+I J b^{2}\right)^{1 / 2}}\left[\frac{I J^{-1 / 2} \beta^{2}+J^{1 / 2} b^{2}}{\left(I^{2} \beta^{2}+I J b^{2}\right)^{1 / 2}}+\frac{a-b c}{k^{1 / 2}}\right] \\
& \quad-1 .
\end{aligned}
$$

Obviously, $d \Pi_{s}(k) / d k$ decreases as $k$ increases. That is, $\Pi_{s}(k)$ is strictly concave in $k$, and thus the maximizer of profit function $\Pi_{s}(k)$ is uniquely determined by the first-order condition; that is, $d \Pi_{s}(k) / d k=0$. Solving the first-order condition for $k$ yields $\overline{\bar{k}}$ of (36). This, together with $0 \leq k \leq K$, leads to $k^{*}$ of (35). Substituting $k=k^{*}$ of (35) into $x=x^{*}(k)$ of (30) and $y=y^{*}(k)$ of (31), respectively, we can get $x^{*}$ of (37) and $y^{*}$ of (38), respectively. We thus complete the proof of Theorem 7.

Theorem 7 gives the supplier's optimal investment strategy in the decentralized supply chain. Note from (20) and (36) that $\bar{k}>\overline{\bar{k}}$. Then, we can get the following corollary from Theorems 3 and 7.

Corollary 8. (i) If $K \leq \overline{\bar{k}}$, then the optimal total amounts of investment resources in the decentralized and the centralized supply chains are both $K$; that is, $k^{*}=k_{c}^{*}=K$. (ii) If $\overline{\bar{k}}<K<$ $\bar{k}$, then the optimal total amount of investment resources in the decentralized supply chain is $\overline{\bar{k}}$, that is, $k^{*}=\overline{\bar{k}}$, and the optimal total amount of investment resources in the centralized supply chain is $K$, that is, $k_{c}^{*}=K$. (iii) If $K \geq \bar{k}$, then the optimal total amount of investment resources in the decentralized supply chain is $\overline{\bar{k}}$, that is, $k^{*}=\overline{\bar{k}}$, and the optimal total amount of investment resources in the centralized supply chain is $\bar{k}$, that is, $k_{c}^{*}=\bar{k}$.

Proof of Corollary 8. Corollary 8 can be obtained directly from Theorems 3 and 7 . We thus complete the proof of Corollary 8.

From Corollary 8, we can see that $k^{*} \leq k_{c}^{*}$. Furthermore, when $K>\overline{\bar{k}}$, we have $k^{*}<k_{c}^{*}$. That is, the optimal total amount of investment resources in the decentralized supply 
chain is strictly lower than that in the centralized supply chain. This reveals the following important managerial insight: when $K>\overline{\bar{k}}$, the additional investment $k_{c}^{*}-k^{*}$ will benefit the entire supply chain but will cause detriment to the supplier's profit. The reason is as follows: in the decentralized supply chain, the benefit caused by the investment is shared by the supplier and the manufacturer, but investment cost is undertaken by the supplier independently, and thus decentralization of decision-making will affect the enthusiasm of the supplier to make investment.

Second, because the ratios of resources invested to achieve cost reduction and quality improvement are identical in the centralized supply chain and decentralized supply chain, that is, $I\left(x^{*}\right)^{2} / J\left(y^{*}\right)^{2}=I\left(x_{c}^{*}\right)^{2} / J\left(y_{c}^{*}\right)^{2}$ (see the discussion following Theorem 6), we can conclude from Corollary 8 that $x^{*} \leq x_{c}^{*}$ and $y^{*} \leq y_{c}^{*}$, for any $K \geq$ 0 . Moreover, when $K>\overline{\bar{k}}$, resources invested in cost reduction and those invested in quality improvement in the decentralized supply chain are both strictly lower than those in the centralized supply chain.

From Lemmas 1 and 4 and Theorems 3 and 7, we can make the following corollary.

Corollary 9. The optimal retail price in the centralized supply chain may or may not be lower than that in the decentralized supply chain.

Proof of Corollary 9. First, we can know from $p_{c}^{*}(x, y)$ of $(7)$, $x_{c}^{*}$ of (21), and $y_{c}^{*}$ of (22) that the optimal retail price in the centralized supply chain is given by

$$
p_{c}^{*}\left(x_{c}^{*}, y_{c}^{*}\right)=\frac{a+\beta y_{c}^{*}+b\left(c-x_{c}^{*}\right)}{2 b} .
$$

Second, we can know from $p^{*}(w, x, y)$ of $(24), w^{*}(x, y)$ of (27), $x^{*}$ of (30), and $y^{*}$ of (31) that the optimal retail price in the decentralized supply chain is given by

$$
p^{*}\left(w^{*}(x, y), x^{*}, y^{*}\right)=\frac{3\left(a+\beta y^{*}\right)+b\left(c-x^{*}\right)}{4 b} \text {. }
$$

Because $x^{*} \leq x_{c}^{*}$ and $y^{*} \leq y_{c}^{*}$, then $p_{c}^{*}\left(x_{c}^{*}, y_{c}^{*}\right)$ may or may not be lower than $p^{*}\left(w^{*}(x, y), x^{*}, y^{*}\right)$. We thus complete the proof of Corollary 9 .

Some current studies on decentralized supply chains state that, due to the fact that the well known double marginalization [29] exists in decentralized supply chains, the optimal retail price in the decentralized setting is higher than that in the centralized setting (see, e.g., $[30,31]$ ). Our finding shows that when the upstream supplier makes investments to reduce its production cost and improve its product quality, the optimal retail price in the decentralized setting may or may not be lower than that in the centralized setting. The reason for our finding can be explained as follows: when the production cost of the product is reduced by the investment, the supplier will charge a lower wholesale price for its product, and then the manufacturer with a lower procurement cost will set a lower retail price for the product.
That is, the investment can increase the retail price. Second, when the product quality is improved by the investment, the supplier will charge a higher wholesale price for its product, and then the manufacturer with a higher procurement cost will set a higher retail price for the product. That is, the investment can decrease the retail price. As a result, it is not clear how the retail price changes with respect to the investment level.

Corollaries 8 and 9 show that both the investment and the retail price decisions in the decentralized setting may deviate from those in the centralized setting. This, together with the fact that the profit of the entire supply chain is uniquely determined by the investment and retail price decisions in our model, implies that such deviations will lead to a loss in the supply chain profit.

\section{Supply Chain Coordination}

In Section 4, we have shown that decentralization of decisionmaking may be detrimental to the profit of the entire supply chain. In this section, we develop a coordination mechanism, aiming to enable the optimal investment and retail price decisions to be chosen by the supplier and the manufacturer in the decentralized supply chain. In addition to the wholesale price in the decentralized supply chain, the developed mechanism contains another three policies: sharing of revenue, sharing of cost reduction investment cost, and sharing of quality improvement investment cost. For convenience, we denote the developed mechanism as $\{w, \phi, \varphi, \psi\}$.

(a) The wholesale price means that the manufacturer pays $w$ to the supplier for each unit of product purchased.

(b) The sharing of revenue means that the manufacturer shares $\phi$ percentage of its sales revenue to the supplier, $0<\phi<1$.

(c) The sharing of cost reduction investment cost means that the manufacturer shares $\varphi$ percentage of the supplier's investment cost for achieving cost reduction, $0<\varphi<1$.

(d) The sharing of quality improvement investment cost means that the manufacturer shares $\psi$ percentage of the supplier's investment cost for achieving quality improvement, $0<\psi<1$.

Under mechanism $\{w, \phi, \varphi, \psi\}$, the total transfer payment from the manufacturer to the supplier can be expressed as

$$
\begin{aligned}
T(w, \phi, \varphi, \psi)= & w(a-b p+\beta y)+\phi p(a-b p+\beta y) \\
& +\varphi I x^{2}+\psi J y^{2} .
\end{aligned}
$$

The first term in (42) is the payment caused by the wholesale price policy, the second term is the payment caused by the revenue-sharing policy, and the last two terms are the payments caused by investment cost-sharing policy. 
Accordingly, the profit functions of the supplier and the manufacturer under mechanism $\{w, \phi, \varphi, \psi\}$ can be expressed, respectively, as follows:

$$
\begin{aligned}
\Pi_{T, s}(w, \phi, \varphi, \psi)= & -(c-x)(a-b p+\beta y)-I x^{2} \\
& -J y^{2}+T(w, \phi, \varphi, \psi) \\
= & {[w-(c-x)+\phi p](a-b p+\beta y) } \\
& -(1-\varphi) I x^{2}-(1-\psi) J y^{2}, \\
\Pi_{T, m}(w, \phi, \varphi, \psi)= & p(a-b p+\beta y)-T(w, \phi, \varphi, \psi) \\
= & {[(1-\phi) p-w](a-b p+\beta y) } \\
& -\varphi I x^{2}-\psi J y^{2} .
\end{aligned}
$$

According to (43) and (44), we have the following theorem.

Theorem 10. Under mechanism $\{w, \phi, \varphi, \psi\}$, let

$$
\begin{aligned}
& w=w(\phi)=(1-\phi)(c-x), \\
& \varphi=\varphi(\phi)=1-\phi, \\
& \psi=\psi(\phi)=1-\phi .
\end{aligned}
$$

Then, for any given $0<\phi<1$, both $\Pi_{T, s}(w, \phi, \varphi, \psi)$ of (43) and $\Pi_{T, m}(w, \phi, \varphi, \psi)$ of (44) achieve their maxima only at $\left(x_{c}^{*}, y_{c}^{*}, p_{c}^{*}\right)$. That is, the decentralized supply chain is coordinated.

Proof of Theorem 10. Substituting (45) into $\Pi_{T, s}(w, \phi, \varphi, \psi)$ of (43) and $\Pi_{T, m}(w, \phi, \varphi, \psi)$ of (44), respectively, the profit functions of the two players under mechanism $\{w(\phi), \phi, \varphi(\phi), \psi(\phi)\}$ can be rewritten, respectively, as follows:

$$
\begin{aligned}
& \Pi_{T, s}(\phi) \\
& \quad=\phi\left\{[p-(c-x)](a-b p+\beta y)-I x^{2}-J y^{2}\right\} \\
& \quad=\phi \Pi_{c}(p \mid x, y), \\
& \Pi_{T, m}(\phi) \\
& \quad=(1-\phi)\left\{[p-(c-x)](a-b p+\beta y)-I x^{2}-J y^{2}\right\} \\
& \quad=(1-\phi) \Pi_{c}(p \mid x, y) .
\end{aligned}
$$

This, in conjunction with the fact that $\Pi_{c}(p \mid x, y)$ achieves its maximum at $x=x_{c}^{*}, y=y_{c}^{*}$, and $p=p_{c}^{*}$, implies that, for any given $0<\phi<1$, both the supplier and the manufacturer prefer $(x, y, p)=\left(x_{c}^{*}, y_{c}^{*}, p_{c}^{*}\right)$. We thus complete the proof of Theorem 10 .

Theorem 10 shows that the developed mechanism $\{w, \phi, \varphi, \psi\}$ with appropriate parameters can coordinate the decentralized supply chain. Here, we should point out that, in practice, an acceptable coordination mechanism should achieve Pareto improvement; that is, the profit of each player is no worse off and at least one player is strictly better off (see, e.g., [32]). In the rest of this section, we will show that the profit of the coordinated supply chain under the developed mechanism $\{w(\phi), \phi, \varphi(\phi), \psi(\phi)\}$ can be arbitrarily divided between the two players, and hence each of the two players can benefit from the developed mechanism.

By substituting (45), $x=x_{c}^{*}$ of (10), $y=y_{c}^{*}$ of (11), and $p=p_{c}^{*}$ of (7) into $\Pi_{T, s}(w, \phi, \varphi, \psi)$ of (43) and $\Pi_{T, m}(w, \phi, \varphi, \psi)$ of (44), respectively, we can obtain the profits of the supplier and the manufacturer under the developed mechanism $\{w(\phi), \phi, \varphi(\phi), \psi(\phi)\}$, respectively, as follows:

$$
\begin{aligned}
\Pi_{T, s}(\phi) & =\phi \Pi_{c}\left(p_{c}^{*} \mid x_{c}^{*}, y_{c}^{*}\right), \\
\Pi_{T, m}(\phi) & =(1-\phi) \Pi_{c}\left(p_{c}^{*} \mid x_{c}^{*}, y_{c}^{*}\right),
\end{aligned}
$$

where $\Pi_{c}\left(p_{c}^{*} \mid x_{c}^{*}, y_{c}^{*}\right)$ is the optimal profit of the centralized supply chain.

One can see from (47) and (48) that, under the developed mechanism $\{w(\phi), \phi, \varphi(\phi), \psi(\phi)\}$, the supplier's profit increases as the revenue share $\phi$ increases, while the manufacturer's profit decreases as the revenue share $\phi$ increases. In addition, one can easily verify from (47) and (48) that $\lim _{\phi \rightarrow 0} \Pi_{T, s}(\phi)=0, \lim _{\phi \rightarrow 1} \Pi_{T, s}(\phi)=\Pi_{c}\left(p_{c}^{*} \mid x_{c}^{*}, y_{c}^{*}\right)$ and $\lim _{\phi \rightarrow 0} \Pi_{T, m}(\phi)=\Pi_{c}\left(p_{c}^{*} \mid x_{c}^{*}, y_{c}^{*}\right), \lim _{\phi \rightarrow 1} \Pi_{T, m}(\phi)=$ 0 . Consequently, the profit of the coordinated supply chain under the developed mechanism $\{w(\phi), \phi, \varphi(\phi), \psi(\phi)\}$ can be arbitrarily divided between the supplier and the manufacturer, and the parameter $\phi$ which can lead to Pareto improvement must exist. The result is summarized as the following corollary.

Corollary 11. If the revenue share $\phi$ satisfies

$$
\frac{\Pi_{s}^{*}}{\Pi_{c}\left(p_{c}^{*} \mid x_{c}^{*}, y_{c}^{*}\right)}<\phi<1-\frac{\Pi_{m}^{*}}{\Pi_{c}\left(p_{c}^{*} \mid x_{c}^{*}, y_{c}^{*}\right)},
$$

then both the supplier and the manufacturer can benefit from participating in the coordination mechanism $\{w(\phi), \phi, \varphi(\phi), \psi(\phi)\}$; that is, $\Pi_{T, s}(\phi)>\Pi_{s}^{*}$ and $\Pi_{T, m}(\phi)>$ $\Pi_{m}^{*}$, where $\Pi_{s}^{*}$ and $\Pi_{m}^{*}$ represent the optimal profits earned by the supplier and the manufacturer in the decentralized supply chain without participating in the coordination mechanism, respectively.

Proof of Corollary 11. (i) Solving $\Pi_{T, s}(\phi)=\phi \Pi_{c}\left(p_{c}^{*} \mid\right.$ $\left.x_{c}^{*}, y_{c}^{*}\right)>\Pi_{s}^{*}$ yields $\phi>\Pi_{s}^{*} / \Pi_{c}\left(p_{c}^{*} \mid x_{c}^{*}, y_{c}^{*}\right)$. (ii) Solving $\Pi_{T, m}(\phi)=(1-\phi) \Pi_{c}\left(p_{c}^{*} \mid x_{c}^{*}, y_{c}^{*}\right)>\Pi_{m}^{*}$ yields $\phi<1-$ $\Pi_{m}^{*} / \Pi_{c}\left(p_{c}^{*} \mid x_{c}^{*}, y_{c}^{*}\right)$. Combining (i) and (ii), we complete the proof of Corollary 11.

Corollary 11 provides a feasible region for the revenue share $\phi$, such that each of the two players can benefit from participating in the coordination mechanism. The feasible region reveals that the supplier has an incentive to increase the revenue share $\phi$, but the manufacturer has an incentive to decrease the revenue share $\phi$. The manufacturer's participation condition $\Pi_{T, m}(\phi)>\Pi_{m}^{*}$ indicates an upper bound of the revenue share $\phi$, that is, $\phi<1-\Pi_{m}^{*} / \Pi_{c}\left(p_{c}^{*} \mid x_{c}^{*}, y_{c}^{*}\right)$, and the supplier's participation condition $\Pi_{T, s}(\phi)>\Pi_{s}^{*}$ indicates 
a lower bound of the revenue share $\phi$, that is, $\phi>\Pi_{s}^{*} / \Pi_{c}\left(p_{c}^{*} \mid\right.$ $\left.x_{c}^{*}, y_{c}^{*}\right)$. Of course, in practice, the actual value of $\phi$ can be determined by the bargaining power between the two players [33].

\section{Conclusion and Future Research}

The existing studies on investments in cost reduction and quality improvement in decentralized supply chains suppose that a supply chain member either makes cost reduction investment or conducts quality improvement investment. This paper considers a supply chain where the upstream supplier can make investment in both cost reduction and quality improvement. Both the centralized supply chain and the decentralized supply chain are explored. Furthermore, the problem of supply chain coordination is investigated. The main results obtained in this paper are summarized as follows:

(1) There exists a unique optimal investment strategy and a unique optimal retail price decision in each of the two supply chains (i.e., centralized supply chain and decentralized supply chain).

(2) The optimal investment strategy in each of the two supply chains requires that the resources be invested in both cost reduction and quality improvement at the same time.

(3) The optimal investment strategy and retail price decision in the decentralized supply chain may deviate from that in the centralized supply chain.

(4) The optimal profit of the decentralized supply chain may be less than that of the centralized supply chain. The mechanism consisting of a wholesale price policy, a revenue-sharing policy, and two investment cost-sharing policies can be used to achieve supply chain coordination by setting mechanism parameters appropriately.

There are some directions for future work. An interesting topic is to investigate the upstream supplier's investment problem in a supply chain with uncertain demand. It is also interesting to consider the downstream manufacturer's joint investments in cost reduction and quality improvement.

\section{Conflicts of Interest}

The authors declare that there are no conflicts of interest regarding the publication of this paper.

\section{References}

[1] J. H. Dyer, D. S. Cho, and W. Chu, "Strategic supplier segmentation: the next 'best practice' in supply chain management," California Management Review, no. 2, pp. 57-77, 1998.

[2] C. Shen, L. Wang, and Q. Li, "Optimization of injection molding process parameters using combination of artificial neural network and genetic algorithm method," Journal of Materials Processing Technology, vol. 183, no. 2-3, pp. 412-418, 2007.
[3] S. Lahiri, "Does outsourcing really improve firm performance? Empirical evidence and research agenda," International Journal of Management Reviews, vol. 18, no. 4, pp. 464-497, 2016.

[4] X. Xu, "Optimal price and product quality decisions in a distribution channel," Management Science, vol. 55, no. 8, pp. 1347-1352, 2009.

[5] G. Xie, W. Yue, S. Wang, and K. K. Lai, "Quality investment and price decision in a risk-averse supply chain," European Journal of Operational Research, vol. 214, no. 2, pp. 403-410, 2011.

[6] G. H. Chao, S. M. R. Iravani, and R. C. Savaskan, "Quality improvement incentives and product recall cost sharing contracts," Management Science, vol. 55, no. 7, pp. 1122-1138, 2009.

[7] M. Seifbarghy, K. Nouhi, and A. Mahmoudi, "Contract design in a supply chain considering price and quality dependent demand with customer segmentation," International Journal of Production Economics, vol. 167, pp. 108-118, 2015.

[8] J. Chen, L. Liang, D.-Q. Yao, and S. Sun, "Price and quality decisions in dual-channel supply chains," European Journal of Operational Research, vol. 259, no. 3, pp. 935-948, 2017.

[9] J. Yu and S. Ma, "Impact of decision sequence of pricing and quality investment in decentralized assembly system," Journal of Manufacturing Systems, vol. 32, no. 4, pp. 664-679, 2013.

[10] W. Li and J. Chen, "Backward integration strategy in a retailer Stackelberg supply chain," Omega.

[11] J. Chen, L. Liang, and F. Yang, "Cooperative quality investment in outsourcing," International Journal of Production Economics, vol. 162, pp. 174-191, 2015.

[12] K. Zhu, R. Q. Zhang, and F. Tsung, "Pushing quality improvement along supply chains," Management Science, vol. 53, no. 3, pp. 421-436, 2007.

[13] P. Ma, H. Wang, and J. Shang, "Supply chain channel strategies with quality and marketing effort-dependent demand," International Journal of Production Economics, vol. 144, no. 2, pp. 572581, 2013.

[14] P. Ma, H. Wang, and J. Shang, "Contract design for twostage supply chain coordination: integrating manufacturerquality and retailer-marketing efforts," International Journal of Production Economics, vol. 146, no. 2, pp. 745-755, 2013.

[15] D. Yang and T. Xiao, "Coordination of a supply chain with lossaverse consumers in service quality," International Journal of Production Research, vol. 55, no. 12, pp. 3411-3430, 2017.

[16] M. Usta, F. Erhun, and W. H. Hausman, "Supply licensing when the manufacturer strategically commits to invest in R\&D," Naval Research Logistics, vol. 61, no. 4, pp. 341-350, 2014.

[17] Z. Ge, Q. Hu, and Y. Xia, "Firms' R\&D cooperation behavior in a supply Chain," Production and Operations Management, vol. 23, no. 4, pp. 599-609, 2014.

[18] S. Banerjee and P. Lin, "Vertical research joint ventures," International Journal of Industrial Organization, vol. 19, no. 1-2, pp. 285-302, 2001.

[19] S. Banerjee and P. Lin, "Downstream R\&D, raising rivals' costs, and input price contracts," International Journal of Industrial Organization, vol. 21, no. 1, pp. 79-96, 2003.

[20] F. Bernstein and A. G. Kök, "Dynamic cost reduction through process improvement in assembly networks," Management Science, vol. 55, no. 4, pp. 552-567, 2009.

[21] F. Bernstein, A. Gürhan Kök, and A. Meca, "Cooperation in assembly systems: the role of knowledge sharing networks," European Journal of Operational Research, vol. 240, no. 1, pp. 160-171, 2015. 
[22] H. Fu, Y. Ma, and X. Cai, "Downstream firm's investment with equity holding in decentralized assembly systems," Omega.

[23] L. Mu and X. Cui, "An experimental research on governance mechanisms for cost of value chain," Nankai Business Review International, vol. 3, no. 2, pp. 199-218, 2012.

[24] A. Ishii, "Cooperative R\&D between vertically related firms with spillovers," International Journal of Industrial Organization, vol. 22, no. 8-9, pp. 1213-1235, 2004.

[25] H. Li, Y. Wang, R. Yin, T. J. Kull, and T. Y. Choi, "Target pricing: demand-side versus supply-side approaches," International Journal of Production Economics, vol. 136, no. 1, pp. 172-184, 2012.

[26] C. d'Aspremont and A. Jacquemin, "Cooperative and noncooperative R\&D in duopoly with spillovers," The American Economic Review, vol. 78, no. 5, pp. 1133-1137, 1988.

[27] D. Ghosh and J. Shah, "A comparative analysis of greening policies across supply chain structures," International Journal of Production Economics, vol. 135, no. 2, pp. 568-583, 2012.

[28] R. D. Banker, I. Khosla, and K. K. Sinha, "Quality and competition," Management Science, vol. 44, no. 9, pp. 1179-1192, 1998.

[29] J. J. Spengler, "Vertical integration and antitrust policy," The Journal of Political Economy, vol. 58, no. 4, pp. 347-352, 1950.

[30] Y. Wang, "Joint pricing-production decisions in supply chains of complementary products with uncertain demand," Operations Research, vol. 54, no. 6, pp. 1110-1127, 2006.

[31] A. David and E. Adida, "Competition and coordination in a two-channel supply chain," Production and Operations Management, vol. 24, no. 8, pp. 1358-1370, 2015.

[32] G. P. Cachon, "Supply chain coordination with contracts," Handbooks in Operations Research and Management Science, vol. 11, pp. 227-340, 2003.

[33] H. Fu, Y. Ma, D. Ni, and X. Cai, "Coordinating a decentralized hybrid push - pull assembly system with unreliable supply and uncertain demand," Annals of Operations Research, pp. 1-21, 2015. 


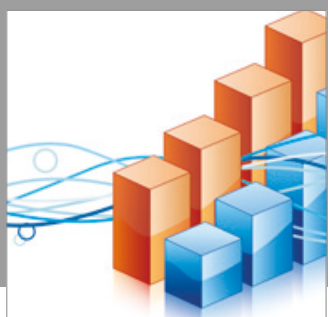

Advances in

Operations Research

vatersals

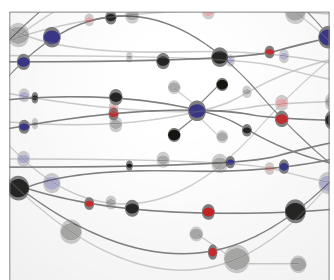

\section{The Scientific} World Journal
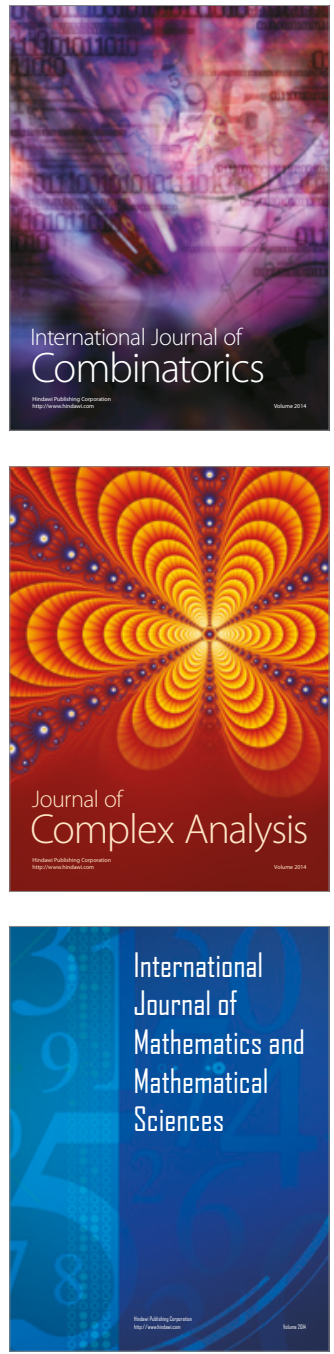
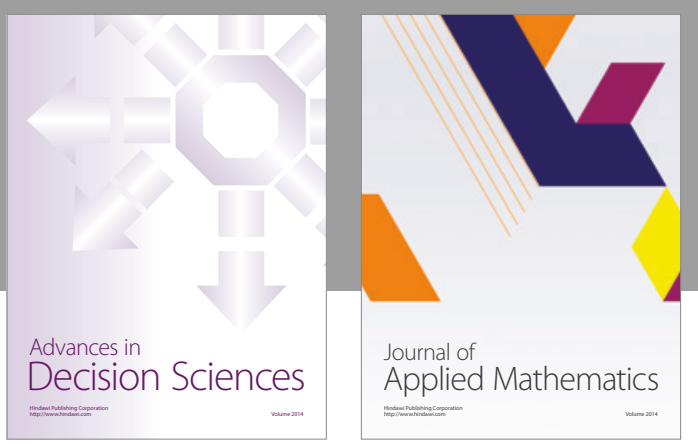

Algebra

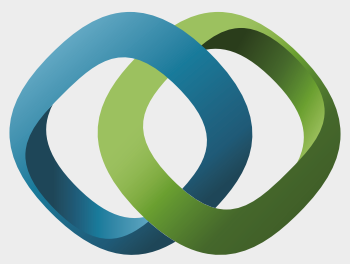

\section{Hindawi}

Submit your manuscripts at

https://www.hindawi.com
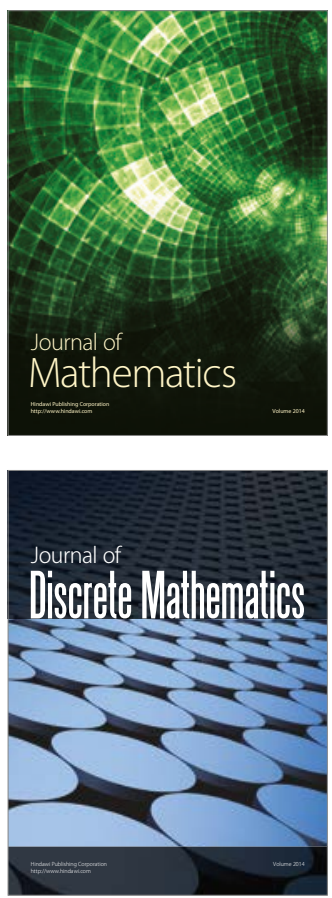

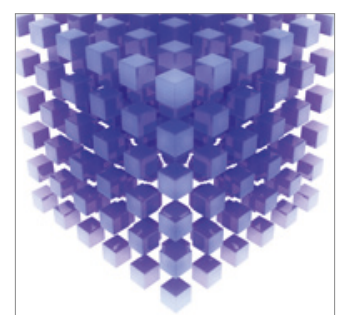

Mathematical Problems in Engineering
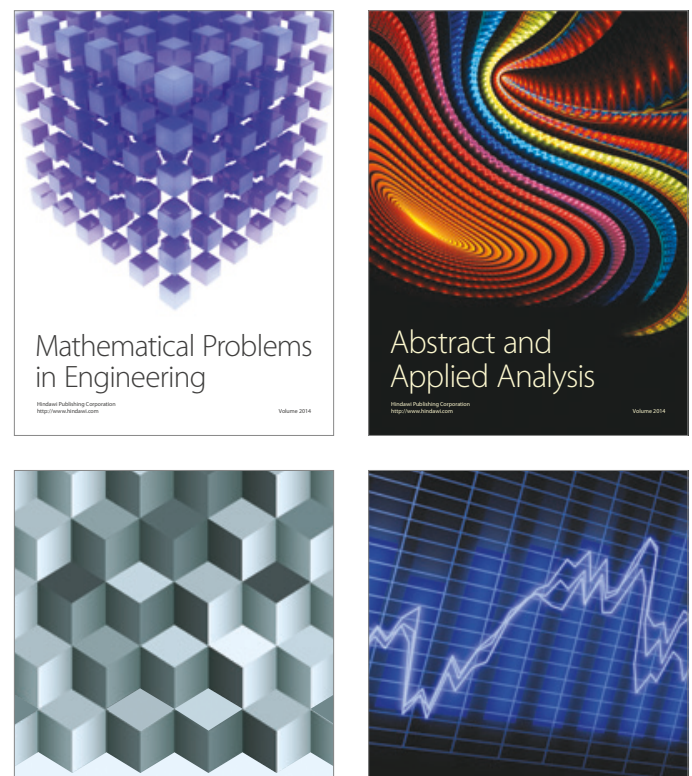

Journal of

Function Spaces

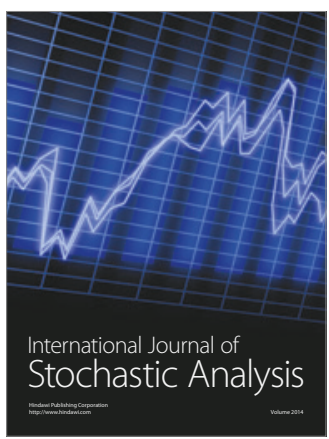

Probability and Statistics
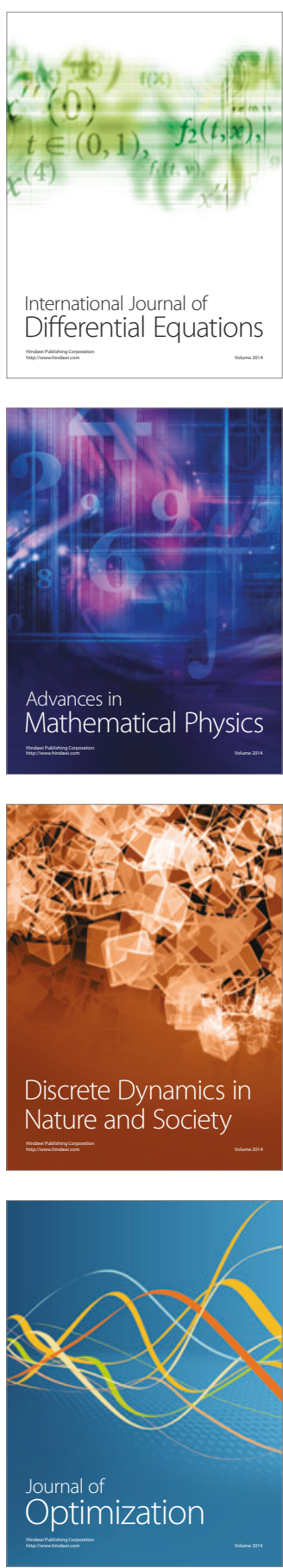